\title{
A nine-node quadrilateral FCBI element for incompressible fluid flows
}

\author{
Haruhiko Kohno ${ }^{1}$ and Klaus-Jürgen Bathe ${ }^{2, *, \dagger}$ \\ ${ }^{1}$ Department of Mathematical Sciences, Coventry University, Priory Street, Coventry CV1 5FB, U.K. \\ ${ }^{2}$ Department of Mechanical Engineering, Massachusetts Institute of Technology, 77 Massachusetts Avenue, \\ Cambridge, MA 02139-4307, U.S.A.
}

\begin{abstract}
SUMMARY
We propose a nine-node flow-condition-based interpolation (FCBI) finite element for the analysis of 2D incompressible flows. Emphasis in the FCBI approach is on obtaining an element that is stable and can be used in rather coarse meshes even when considering high Reynolds number flows. The formulation of the element is presented and the results obtained in the solution of some test cases are given. Copyright (C) 2006 John Wiley \& Sons, Ltd.
\end{abstract}

KEY WORDS: incompressible flows; flow-condition-based interpolation; FCBI approach

\section{INTRODUCTION}

While numerical solutions of the Navier-Stokes equations are obtained daily by many engineers and scientists, the more effective solution of fluid flows represents still a major challenge, see for example References [1-4] and the references therein. More stable, more accurate, and computationally more efficient techniques are needed.

An important field is the solution of incompressible fluid flows with structural interactions. Here frequently, the purpose of the analysis is to analyse the structure accurately for some specific quantities but the fluid flow response may not be needed to be highly accurate. The fluid flow must then only be simulated to solve accurately for the fluid forces that act onto the structure, and clearly the use of very coarse meshes to solve for the fluid actions is attractive. Then, to ensure good accuracy in the structural response of interest, like the bending moment at the base of a rotor blade, goal-oriented error measures can be used $[5,6]$.

\footnotetext{
*Correspondence to: Klaus-Jürgen Bathe, Department of Mechanical Engineering, Massachusetts Institute of Technology, 77 Massachusetts Avenue, Cambridge, MA 02139-4307, U.S.A.

†E-mail: kjb@mit.edu

Contract/grant sponsor: Japan Society for the Promotion of Science (JSPS)
}

Copyright (c) 2006 John Wiley \& Sons, Ltd.

Received 2 September 2005

Revised 25 January 2006

Accepted 31 January 2006 
However, in order to be able to use a coarse fluid mesh, it is necessary that the numerical procedure for the fluid flow be stable, even at high Reynolds number flows, and always give a reasonable solution [7,8]. Of course, ideally, the solution should also be obtained using consistent Jacobian matrices, for computational efficiency, and be as accurate as possible for the coarse mesh used.

Towards this aim, we have worked on the development of finite elements with flowcondition-based interpolations (FCBI). These elements use the usual velocity and pressure interpolations for the diffusion and pressure terms, respectively, to satisfy the inf-sup condition for incompressible analysis, and special velocity interpolations, dependent on the element Reynolds numbers, for the advection term. Also, control volumes are used to satisfy local momentum and mass conservation [8-11]. Naturally, these FCBI element formulations are related to earlier proposed techniques $[3,9]$ and we have drawn on these to provide more effective procedures. The FCBI approach is already used widely in the CFD and FSI solution capabilities of ADINA [11] but we continue our research to improve the specific techniques used. In particular, only effective low-order elements are available in ADINA and we are still searching for more effective higher order elements, as these would be useful for error estimation, see Reference [6].

The objective of this paper is to propose a new nine-node element FCBI formulation for two-dimensional fluid flows. We concentrate on the two-dimensional case to present and study the technique but the three-dimensional implementation can directly be achieved. We first present the formulation and then the results of some example solutions.

\section{A NEW FCBI METHOD FOR THE SOLUTION OF THE NAVIER-STOKES EQUATIONS}

In this section, we present a new nine-node element based on the FCBI approach for the analysis of incompressible fluid flows. We first give the mathematical model considered and then present the interpolations used.

\subsection{Governing equations and finite element formulation}

We consider a two-dimensional steady-state flow problem governed by the incompressible Navier-Stokes equations. We assume that the problem is well-posed in the Hilbert spaces $V$ and $P$. The non-dimensional governing equations in conservative form are:

Find the velocity $\mathbf{v}(\mathbf{x}) \in V$ and pressure $p(\mathbf{x}) \in P$ such that

$$
\begin{aligned}
\nabla \cdot \mathbf{v}=0, & \mathbf{x} \in \Omega \\
\nabla \cdot(\mathbf{v v}-\tau)=\mathbf{0}, & \mathbf{x} \in \Omega
\end{aligned}
$$

subject to the boundary conditions

$$
\begin{array}{rr}
\mathbf{v}=\mathbf{v}^{s}, & \mathbf{x} \in \bar{S}_{v} \\
\tau \cdot \mathbf{n} & =\mathbf{f}^{s}, \quad \mathbf{x} \in S_{f}
\end{array}
$$


where $\Omega \in \Re^{2}$ is a domain with the boundary $S=\bar{S}_{v} \cup S_{f}\left(S_{v} \cap S_{f}=\emptyset\right), \tau$ is the stress tensor defined as

$$
\tau=\tau(\mathbf{v}, p)=-p \mathbf{I}+\frac{1}{R e}\left\{\nabla \mathbf{v}+(\nabla \mathbf{v})^{\mathrm{T}}\right\}
$$

with the identity tensor I and the Reynolds number $R e, \mathbf{v}^{s}$ is the prescribed velocity on the boundary $\bar{S}_{v}, \mathbf{f}^{s}$ is the prescribed traction on the boundary $S_{f}$, and $\mathbf{n}$ is the unit normal vector to the boundary.

For the finite element solution, we use a Petrov-Galerkin variational formulation with subspaces $U_{h}, V_{h}$ and $W_{h}$ of $V$, and $P_{h}$ and $Q_{h}$ of $P$ of the problem in Equations (1)-(4). The formulation for the numerical solution is:

Find $\mathbf{u} \in U_{h}, \mathbf{v} \in V_{h}$ and $p \in P_{h}$ such that for all $w \in W_{h}$ and $q \in Q_{h}$ :

$$
\begin{aligned}
\int_{\Omega} w \nabla \cdot(\mathbf{u v}-\tau(\mathbf{u}, p)) \mathrm{d} \Omega & =\mathbf{0} \\
\int_{\Omega} q \nabla \cdot \mathbf{u} \mathrm{d} \Omega & =0
\end{aligned}
$$

The trial functions in $U_{h}$ and $P_{h}$ are the conventional functions of finite element interpolations for velocity and pressure, respectively, used to satisfy the inf-sup condition of incompressible analysis [12]. In order to provide also stability regarding the advection term (the upwinding effect), the trial functions in $V_{h}$ are defined considering the flow conditions. The weight functions in the spaces $W_{h}$ and $Q_{h}$ are step functions, which enforce the local conservation of momentum and mass, respectively.

\subsection{The nine-node element}

Based on the original FCBI methods $[7,8]$, we propose in this section a new FCBI procedure to reach a more effective nine-node element. As for the other FCBI methods, the functions introducing the upwinding effect are spatially isotropic and are established by incorporating the flow conditions given by solutions of advection-diffusion equations. A nine-node element was already proposed in Reference [7], but the flow-condition-based functions in that earlier contribution are quite different from those used in the present paper (see Remark 3) and are used as weight functions for the convection and diffusion terms. Also, control volumes are not used. Another nine-node element is discussed in Reference [8] using control volumes as in the present paper, but four 'sub-elements' per nine-node element are used. Hence the velocity interpolations do not naturally approach the usual biquadratic interpolations as the flow Reynolds number decreases. On the other hand, the nine-node element we discuss in the present paper does have this desirable property, and hence, when the local intensity of fluid flow (measured by the element Reynolds numbers) becomes small, the discretization scheme gives second-order accuracy.

Figure 1 shows a nine-node element in which the velocity is defined through nine nodes, the local node numbers $1-9$, while the pressure is defined through the four corner nodes, the local node numbers $1-4$, in order to satisfy the inf-sup condition. If the usual Lagrangian interpolation functions are used, this element is of course the Taylor-Hood element, or 


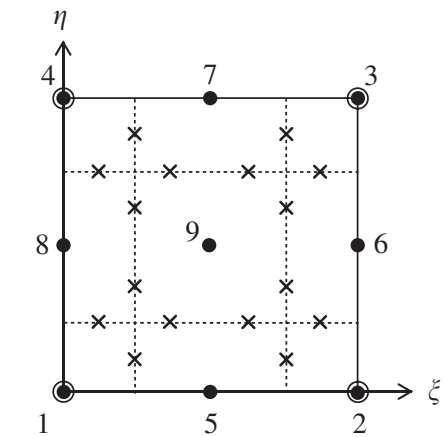

(a)

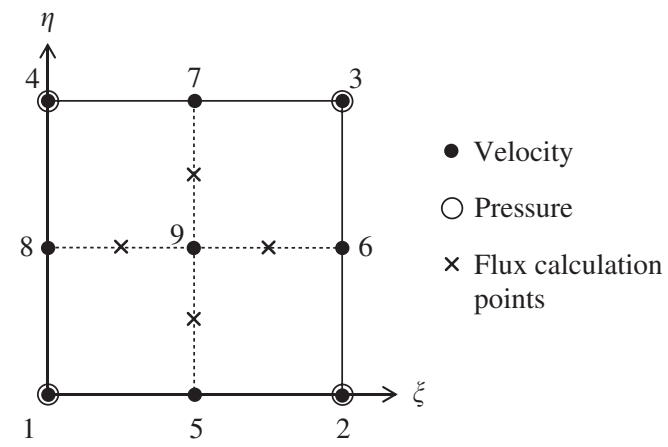

(b)

Figure 1. A nine-node element and segmentation for the control volumes: (a) segments in the space $W_{h}$; and (b) segments in the space $Q_{h}$.

Q9-Q4 or 9/4-c element [12]. The weight functions in the space $W_{h}$ are the step functions as in References [8-11], for example for node 9 defined as

$$
h_{9}^{w}= \begin{cases}1, & (\xi, \eta) \in\left[\frac{1}{4}, \frac{3}{4}\right] \times\left[\frac{1}{4}, \frac{3}{4}\right] \\ 0 & \text { else }\end{cases}
$$

Similarly, the weight functions in the space $Q_{h}$ are the step functions, for example for node 1 defined as

$$
h_{1}^{q}= \begin{cases}1, & (\xi, \eta) \in\left[0, \frac{1}{2}\right] \times\left[0, \frac{1}{2}\right] \\ 0 & \text { else }\end{cases}
$$

Therefore, the control volumes corresponding to the spaces $W_{h}$ and $Q_{h}$ are as shown in Figures 1(a) and (b), respectively. The flux is calculated with the interpolated values at the centre of the control volume sides, which are divided equally into eight per control volume in the calculation space.

The velocity $\mathbf{u}$ and the pressure $p$ are, respectively, calculated with the trial functions in $U_{h}$ and $P_{h}$ as follows:

$$
\begin{aligned}
& \mathbf{u}=h_{i}^{u} \mathbf{v}_{i} \\
& p=h_{i}^{p} p_{i}
\end{aligned}
$$

where $\mathbf{v}_{i}$ and $p_{i}$ are the nodal velocity and pressure variables, respectively. The trial functions in $U_{h}$ are defined as

$$
\left[\begin{array}{lll}
h_{1}^{u} & h_{4}^{u} & h_{8}^{u} \\
h_{2}^{u} & h_{3}^{u} & h_{6}^{u} \\
h_{5}^{u} & h_{7}^{u} & h_{9}^{u}
\end{array}\right]=\mathbf{h}(\xi) \mathbf{h}^{\mathrm{T}}(\eta)
$$

where

$$
\mathbf{h}^{\mathrm{T}}(y)=\left[h_{1}(y), h_{2}(y), h_{3}(y)\right]=[(1-2 y)(1-y), y(2 y-1), 4 y(1-y)] \quad(y=\xi, \eta \text { with } 0 \leqslant \xi, \eta \leqslant 1)
$$




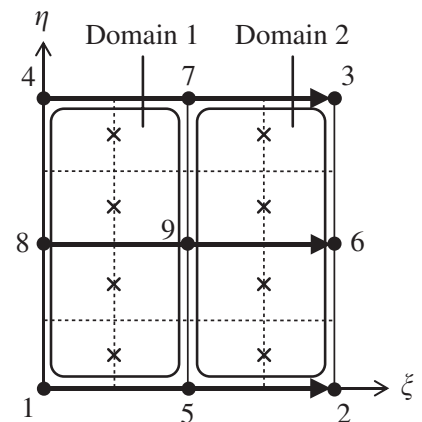

(a)

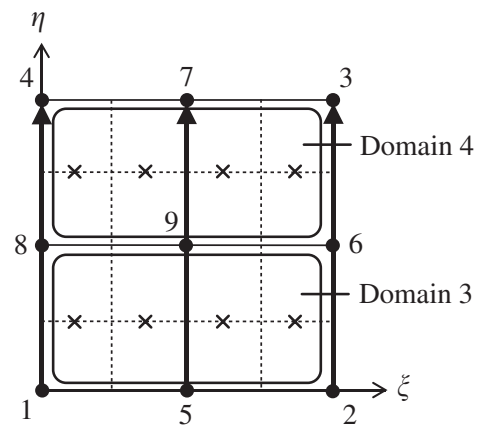

(b)

Figure 2. Four domains in an element considered in the trial functions in $V_{h}$ : (a) FCBI in the $\xi$ direction; and (b) FCBI in the $\eta$ direction.

which represents the biquadratic interpolation. On the other hand, the trial functions in $P_{h}$ are given by

$$
\left[\begin{array}{ll}
h_{1}^{p} & h_{4}^{p} \\
h_{2}^{p} & h_{3}^{p}
\end{array}\right]=\mathbf{h}^{*}(\xi) \mathbf{h}^{*^{\mathrm{T}}}(\eta)
$$

where $\mathbf{h}^{*^{\mathrm{T}}}(y)=[1-y, y] \quad(y=\xi, \eta$ with $0 \leqslant \xi, \eta \leqslant 1)$, which represents the bilinear interpolation. The interpolation functions for pressure are the same as those in the original FCBI methods $[7,8]$.

The trial functions in $V_{h}$ are defined by employing the flow conditions on the element sides and centre lines. As in the FCBI method for triangular grids [10], we consider different interpolation functions for the velocity component vectors $\mathbf{v}_{\|}$and $\mathbf{v}_{\perp}$, the directions of which are, respectively, parallel and perpendicular to each side or centre line of the element. Then, the velocity $\mathbf{v}$ in the advection term is calculated as follows:

$$
\begin{aligned}
\mathbf{v} & =h_{i \|}^{v} \mathbf{v}_{i \|}+h_{i \perp}^{v} \mathbf{v}_{i \perp} \\
& =h_{i}^{v} \mathbf{v}_{i \|}+h_{i}^{u} \mathbf{v}_{i \perp}
\end{aligned}
$$

where $\mathbf{v}_{i \|}$ and $\mathbf{v}_{i \perp}$ are the nodal velocity component vectors that are parallel and perpendicular to the corresponding side or centre line, respectively. The interpolation functions for the perpendicular component vector correspond to the trial functions in $U_{h}$ and can be interpreted as a particular case of the functions for the parallel component vector (see Remark 1). By incorporating the flow conditions into the interpolation, the functions $h_{i}^{v}\left(=h_{i \|}^{v}\right)$ are defined in the four domains shown in Figure 2 as follows (for a detailed derivation, see Appendix A): In the domain 1:

$$
\left[\begin{array}{lll}
h_{1}^{v} & h_{4}^{v} & h_{8}^{v} \\
h_{2}^{v} & h_{3}^{v} & h_{6}^{v} \\
h_{5}^{v} & h_{7}^{v} & h_{9}^{v}
\end{array}\right]=\left[\mathbf{h}\left(x^{15}\right), \mathbf{h}\left(x^{47}\right), \mathbf{h}\left(x^{89}\right)\right] \operatorname{diag}\left(h_{1}(\eta), h_{2}(\eta), h_{3}(\eta)\right)
$$


with

$$
x^{i j}=\frac{1}{2} \frac{\mathrm{e}^{R e_{i j}^{e}(2 \xi)}-1}{\mathrm{e}^{R e_{i j}^{e}}-1}
$$

In the domain 2:

$$
\left[\begin{array}{lll}
h_{1}^{v} & h_{4}^{v} & h_{8}^{v} \\
h_{2}^{v} & h_{3}^{v} & h_{6}^{v} \\
h_{5}^{v} & h_{7}^{v} & h_{9}^{v}
\end{array}\right]=\left[\mathbf{h}\left(x^{52}\right), \mathbf{h}\left(x^{73}\right), \mathbf{h}\left(x^{96}\right)\right] \operatorname{diag}\left(h_{1}(\eta), h_{2}(\eta), h_{3}(\eta)\right)
$$

with

$$
x^{i j}=\frac{1}{2}\left(\frac{\mathrm{e}^{R e_{i j}^{e}(2 \xi-1)}-1}{\mathrm{e}^{R e_{i j}^{e}}-1}+1\right)
$$

In the domain 3:

$$
\left[\begin{array}{lll}
h_{1}^{v} & h_{2}^{v} & h_{5}^{v} \\
h_{4}^{v} & h_{3}^{v} & h_{7}^{v} \\
h_{8}^{v} & h_{6}^{v} & h_{9}^{v}
\end{array}\right]=\left[\mathbf{h}\left(x^{18}\right), \mathbf{h}\left(x^{26}\right), \mathbf{h}\left(x^{59}\right)\right] \operatorname{diag}\left(h_{1}(\xi), h_{2}(\xi), h_{3}(\xi)\right)
$$

with

$$
x^{i j}=\frac{1}{2} \frac{\mathrm{e}^{R e_{i j}^{e}(2 \eta)}-1}{\mathrm{e}^{R e_{i j}^{e}}-1}
$$

In the domain 4:

$$
\left[\begin{array}{lll}
h_{1}^{v} & h_{2}^{v} & h_{5}^{v} \\
h_{4}^{v} & h_{3}^{v} & h_{7}^{v} \\
h_{8}^{v} & h_{6}^{v} & h_{9}^{v}
\end{array}\right]=\left[\mathbf{h}\left(x^{84}\right), \mathbf{h}\left(x^{63}\right), \mathbf{h}\left(x^{97}\right)\right] \operatorname{diag}\left(h_{1}(\xi), h_{2}(\xi), h_{3}(\xi)\right)
$$

with

$$
x^{i j}=\frac{1}{2}\left(\frac{\mathrm{e}^{R e_{i j}^{e}(2 \eta-1)}-1}{\mathrm{e}^{R e_{i j}^{e}}-1}+1\right)
$$

where the subscripts and superscripts $i$ and $j$ represent the local node numbers. In Equations (15), (17), (19) and (21), $\mathbf{h}\left(x^{i j}\right)$ is written in the same form as in Equation (12). The element Reynolds numbers $R e_{i j}^{e}$ are defined as follows:

$$
\operatorname{Re} e_{i j}^{e}=\operatorname{Re} \overline{\mathbf{v}} \cdot \Delta \mathbf{x}_{i j}
$$

with

$$
\overline{\mathbf{v}}=\frac{1}{6}\left(\mathbf{v}_{r}+4 \mathbf{v}_{s}+\mathbf{v}_{t}\right), \quad \Delta \mathbf{x}_{i j}=\mathbf{x}_{j}-\mathbf{x}_{i}
$$

where $\overline{\mathbf{v}}$ is the average velocity on the corresponding side or centre line, which is obtained with two nodal velocities $\mathbf{v}_{r}, \mathbf{v}_{t}$ at the ends and a nodal velocity $\mathbf{v}_{s}$ at the centre; $\mathbf{x}_{i}$ and $\mathbf{x}_{j}$ 

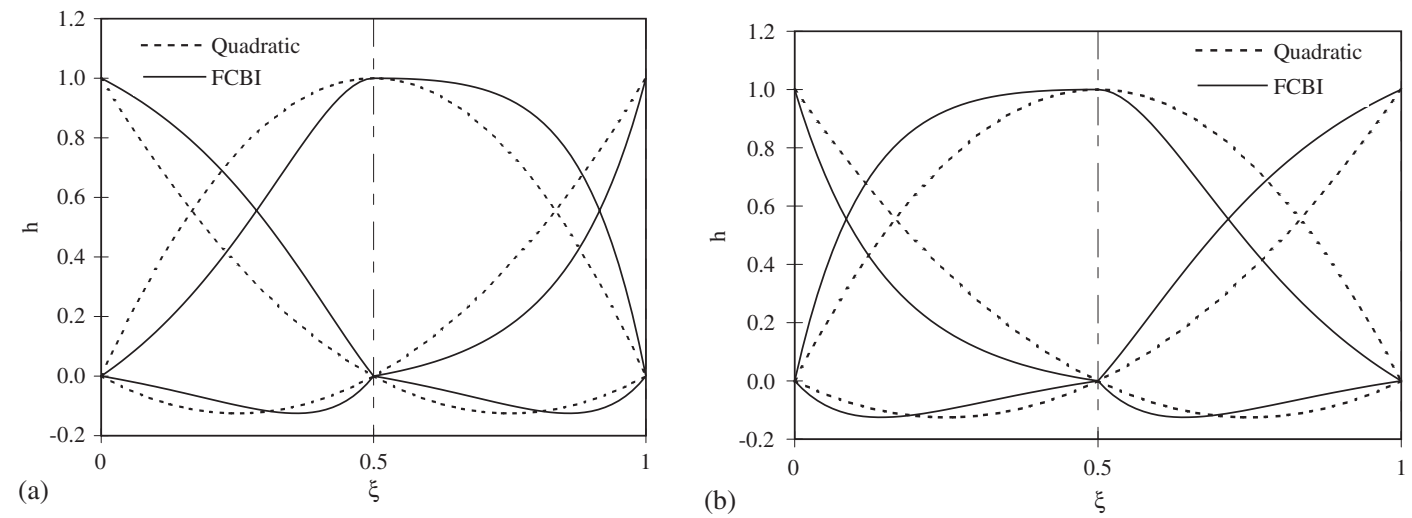

Figure 3. Comparison of the profiles of the functions between quadratic interpolation and FCBI: (a) $R e^{e}=2$; and (b) $R e^{e}=-2$.

are the position vectors at the nodes. The newly developed trial functions given above satisfy the requirement $\sum h_{i}^{v}=1$ regardless of the element Reynolds numbers. Moreover, when the absolute value of the element Reynolds number becomes small, the functions in Equations (15), (17), (19) and (21) approach the biquadratic interpolation functions in Equation (12). The profiles of the trial functions $h_{i}^{v}$ for $R e^{e}= \pm 2$ in the one-dimensional space $(0 \leqslant \xi \leqslant 1)$ are compared with those of quadratic interpolation in Figure 3 . It is seen that the upwinding effect is suitably generated according to the flow direction.

\section{Remark 1}

If the trial functions $h_{i}^{v}$ were also applied to the perpendicular component vector of velocity, the interpolation in Equation (14) would be

$$
\mathbf{v}=h_{i}^{v}\left(\mathbf{v}_{i \|}+\mathbf{v}_{i \perp}\right)=h_{i}^{v} \mathbf{v}_{i}
$$

However, it is more accurate to use different interpolations for the parallel and perpendicular component vectors of velocity because the element Reynolds number in the perpendicular direction can be regarded as infinitesimally small for the scheme used (consequently, the trial functions for the perpendicular component vector are still the quadratic interpolations on the element sides or centre lines). This approach is also mentioned in Reference [10], and the improvement in accuracy is illustrated therein.

\section{Remark 2}

Since the calculation is conducted in the Cartesian co-ordinates, Equation (14) need be converted into a form using the nodal velocity components $v_{i x}$ and $v_{i y}$ corresponding to the $x$ and $y$ directions, respectively. First, we note that the nodal velocity component vectors $\mathbf{v}_{i \|}$ and $\mathbf{v}_{i \perp}$ have the following relations with the components $v_{i x}$ and $v_{i y}$ :

$$
\begin{aligned}
\mathbf{v}_{i \|} & =v_{i \|} \mathbf{e}_{\|}^{j k} \\
& =\left\{v_{i x}\left(e_{\|}^{j k}\right)_{x}+v_{i y}\left(e_{\|}^{j k}\right)_{y}\right\} \mathbf{e}_{\|}^{j k}
\end{aligned}
$$




$$
\begin{aligned}
\mathbf{v}_{i \perp} & =v_{i \perp} \mathbf{e}_{\perp}^{j k} \\
& =\left\{v_{i x}\left(e_{\perp}^{j k}\right)_{x}+v_{i y}\left(e_{\perp}^{j k}\right)_{y}\right\} \mathbf{e}_{\perp}^{j k}
\end{aligned}
$$

with

$$
\begin{aligned}
\mathbf{e}_{\|}^{j k} & =\frac{\Delta \mathbf{x}_{j k}}{\left\|\Delta \mathbf{x}_{j k}\right\|}, \quad \mathbf{e}_{\perp}^{j k}=\mathbf{e}_{x} \times \mathbf{e}_{y} \times \mathbf{e}_{\|}^{j k} \\
\mathbf{e}_{\|}^{j k} \cdot \mathbf{e}_{x} & =\left(e_{\|}^{j k}\right)_{x}, \quad \mathbf{e}_{\|}^{j k} \cdot \mathbf{e}_{y}=\left(e_{\|}^{j k}\right)_{y} \\
\mathbf{e}_{\perp}^{j k} \cdot \mathbf{e}_{x} & =\left(e_{\perp}^{j k}\right)_{x}, \quad \mathbf{e}_{\perp}^{j k} \cdot \mathbf{e}_{y}=\left(e_{\perp}^{j k}\right)_{y}
\end{aligned}
$$

where the subscript or superscript $i, j$ and $k$ represent the local node numbers, $\mathbf{e}_{x}$ and $\mathbf{e}_{y}$ are the unit vectors in the $x$ and $y$ directions, respectively. Then, by substituting Equations (26)-(28) into Equation (14), $v_{x}$ and $v_{y}$ in the four domains are obtained with the nodal velocity components $v_{i x}$ and $v_{i y}$ as follows:

$$
\begin{aligned}
v_{x} & =\mathbf{v} \cdot \mathbf{e}_{x} \\
& =\left(h_{i}^{v} \mathbf{v}_{i \|}+h_{i}^{u} \mathbf{v}_{i \perp}\right) \cdot \mathbf{e}_{x} \\
& =\sum_{i=1}^{9} \alpha_{i x} v_{i x}+\sum_{i=1}^{9} \beta_{i x} v_{i y} \\
v_{y} & =\mathbf{v} \cdot \mathbf{e}_{y} \\
& =\left(h_{i}^{v} \mathbf{v}_{i \|}+h_{i}^{u} \mathbf{v}_{i \perp}\right) \cdot \mathbf{e}_{y} \\
& =\sum_{i=1}^{9} \alpha_{i y} v_{i x}+\sum_{i=1}^{9} \beta_{i y} v_{i y}
\end{aligned}
$$

The coefficients have the following properties:

$$
\sum_{i=1}^{9} \alpha_{i x}=1, \quad \sum_{i=1}^{9} \beta_{i x}=0, \quad \sum_{i=1}^{9} \alpha_{i y}=0, \quad \sum_{i=1}^{9} \beta_{i y}=1
$$

due to the relation $\left(e_{\|}^{j k}\right)_{x}=\left(e_{\perp}^{j k}\right)_{y}$ and $\left(e_{\|}^{j k}\right)_{y}=-\left(e_{\perp}^{j k}\right)_{x}$. Therefore, the requirement $\sum h_{i}^{v}=1$ is still satisfied in both velocity components. Moreover, it is readily confirmed from Equations (29)-(31) that the uniformity condition is also satisfied; if the identical velocity value $\left(v_{x}, v_{y}\right)=\left(v_{a}, v_{b}\right)$ is prescribed at the nine nodes, the interpolated value is also $\left(v_{x}, v_{y}\right)=\left(v_{a}, v_{b}\right)$ at any point in the element. The interpolated values in $v_{x}$ and $v_{y}$ are independent of the directions $\mathbf{e}_{\|}^{j k}$ and $\mathbf{e}_{\perp}^{j k}$ as long as these unit vectors are, respectively, parallel and perpendicular to the side $j-k$.

\section{Remark 3}

It is worth pointing out the difference in the interpolation functions to those proposed for a nine-node element in Reference [7]. Although both functions approach biquadratic interpolation functions when the absolute value of the element Reynolds number decreases, the flow 
conditions are differently evaluated on the element sides and centre lines. The previous FCBI method adopts the following approximate solution:

$$
w(\xi)=w_{3}+a \xi+b\left(\mathrm{e}^{-\kappa \xi}-1\right)
$$

of the one-dimensional advection-diffusion equation with

$$
\kappa=\frac{\operatorname{Re} v_{3} \Delta x}{2}, \quad \Delta x=x_{2}-x_{1}
$$

Here, the nodal values $w_{1}, w_{2}$ and $w_{3}$ are, respectively, defined at $x_{1}, x_{2}$ and $x_{3}\left(x_{1} \leqslant x \leqslant x_{2}\right.$, $\left.x_{3}=\left(x_{1}+x_{2}\right) / 2\right)$, and $v_{3}$ is the velocity at $x_{3}$. The constants $a$ and $b$ are determined by substituting the following boundary conditions into Equation (32): $w_{1}=w(\xi=-1), w_{2}=w(\xi=1)$. Consequently, the following interpolation functions are obtained:

$$
w(\xi)=h_{1}^{w}(\xi) w_{1}+h_{2}^{w}(\xi) w_{2}+h_{3}^{w}(\xi) w_{3}
$$

with

$$
\begin{aligned}
& h_{1}^{w}(\xi)=\frac{\left(\mathrm{e}^{-\kappa \xi}-\xi \mathrm{e}^{-\kappa}\right)-(1-\xi)}{\mathrm{e}^{\kappa}+\mathrm{e}^{-\kappa}-2}, \quad h_{2}^{w}(\xi)=\frac{\left(\mathrm{e}^{-\kappa \xi}+\xi \mathrm{e}^{\kappa}\right)-(1+\xi)}{\mathrm{e}^{\kappa}+\mathrm{e}^{-\kappa}-2} \\
& h_{3}^{w}(\xi)=\frac{(1+\xi) \mathrm{e}^{-\kappa}+(1-\xi) \mathrm{e}^{\kappa}-2 \mathrm{e}^{-\kappa \xi}}{\mathrm{e}^{\kappa}+\mathrm{e}^{-\kappa}-2}
\end{aligned}
$$

Note the interpolation functions in Equation (35) are used as weight (test) functions in the former nine-node FCBI element. When we consider extending the above idea to the interpolation of velocity, it appears to be appropriate to employ the following approximate solution:

$$
v(\xi)=v_{3}+a \xi+b\left(\mathrm{e}^{\kappa \xi}-1\right)
$$

in which ' $-\kappa \xi$ ' in Equation (32) is replaced with ' $\kappa \xi$ ' in order to reverse the profiles of the functions. However, there is one deficiency; the interpolation function can exceed one $\left(h_{3}^{v}>1\right)$ when the absolute value of $\kappa$ is large. For example, $h_{1}^{v}=-0.8002, h_{2}^{v}=0.0498$ and $h_{3}^{v}=1.7504$ at $\xi=0.85$ when $\kappa=20$, and then if $v_{1}=1.0, v_{2}=1.3, v_{3}=1.2$; then $v(\xi=0.85)=1.3650$ $\left(>v_{1}, v_{2}, v_{3}\right)$. The interpolation functions of the proposed nine-node FCBI element never exceed one (see Appendix A).

\section{NUMERICAL EXAMPLES}

In this section, the performance of the proposed nine-node FCBI method is illustrated through some test problems. First we verify the validity of the scheme in an advection-diffusion problem, for which the exact analytical solution exists. Then we analyse the lid-driven flow in a square cavity as a Navier-Stokes flow problem. The full Newton-Raphson method is used to solve the nonlinear equations with the convergence criteria $\max \left(R_{v}\right) \leqslant 10^{-6}$ and $\max \left(R_{p}\right) \leqslant 10^{-6}$ where $R_{v}=\|\Delta \mathbf{v}\| /\|\mathbf{v}\|, R_{p}=|\Delta p| /|p|$. To reach the solutions for higher Reynolds numbers, we use the converged solution of the lower Reynolds number case as an initial condition. 


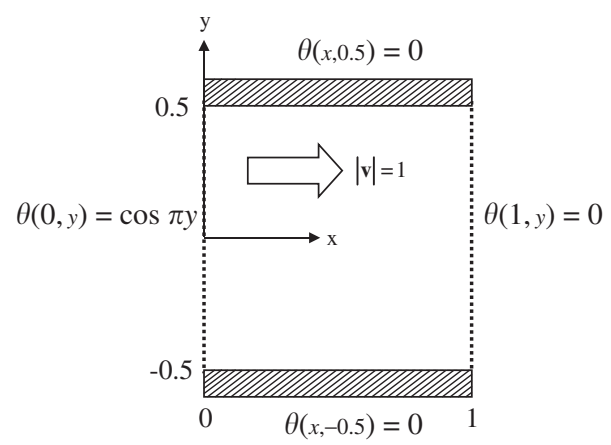

Figure 4. A schematic diagram of the flow problem between parallel plates.
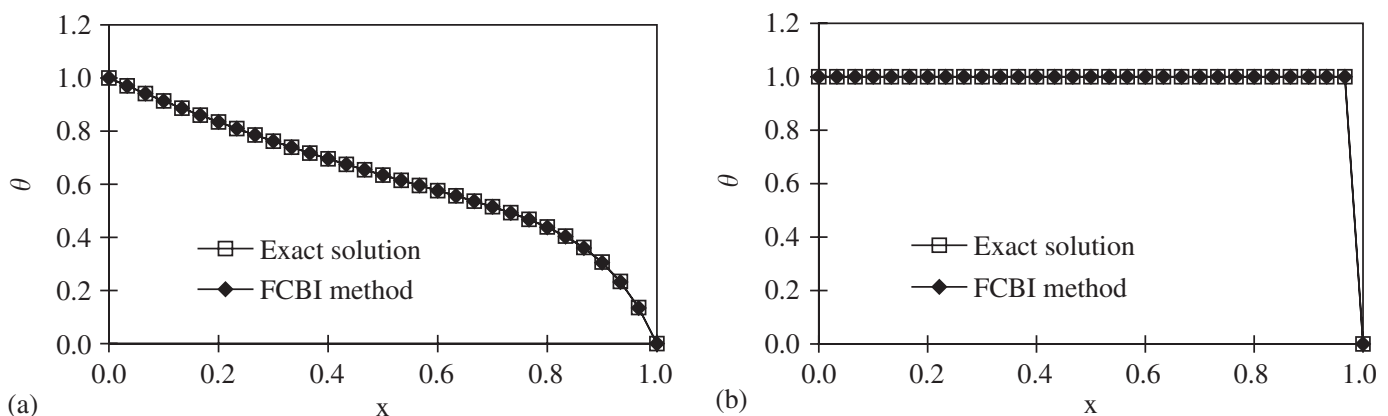

Figure 5. Comparison of temperature values on the centre line: (a) $P e=10$; and (b) $P e=10^{6}$.

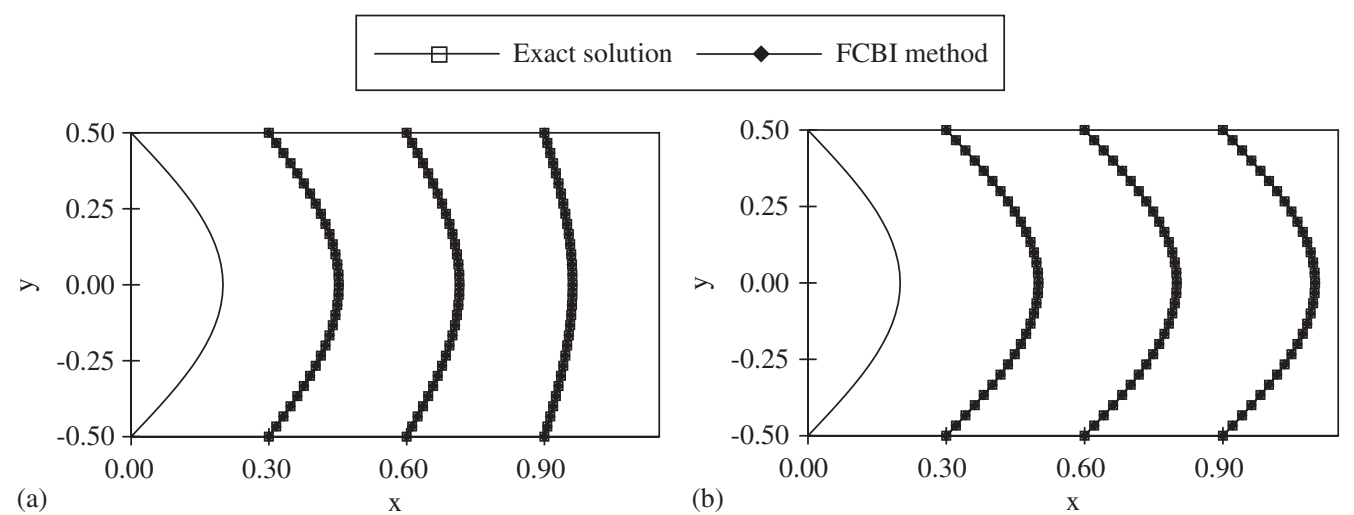

Figure 6. Comparison of temperature profiles on vertical lines: (a) $P e=10$; and (b) $P e=10^{6}$. 


\subsection{Solution of an advection-diffusion temperature problem between parallel plates}

We first solve this problem in order to compare our calculated results with analytical solutions. This temperature problem is a good test problem to assess the stability of the proposed method. Of course, the above scheme is directly applicable (with the temperature now the unknown, element Péclet numbers used, and so on [9]). Figure 4 shows the analytical model of the temperature problem considered with the boundary conditions. When a unit velocity is prescribed in the $x$ direction over the whole domain, the exact steady-state solution for the temperature $\theta$ is

$$
\theta(x, y)=\frac{\cos \pi y}{\mathrm{e}^{a}-\mathrm{e}^{b}}\left(\mathrm{e}^{a+b x}-\mathrm{e}^{b+a x}\right)
$$

with

$$
a=\frac{1}{2}\left(P e+\sqrt{P e^{2}+4 \pi^{2}}\right), \quad b=\frac{1}{2}\left(P e-\sqrt{P e^{2}+4 \pi^{2}}\right)
$$

where $P e$ is the Péclet number.

A mesh of $15 \times 15$ uniform elements is used in this study. Since the temperature is a scalar variable, it is interpolated with only the trial functions $h_{i}^{v}$ in the advection term while the functions $h_{i}^{u}$ are employed for the interpolations in the diffusion term.

Figures 5 and 6 show the comparison of temperature values on the centre line and on vertical lines transverse to the flow for $P e=10$ and $10^{6}$. Good agreement with the exact solutions can be seen in the low and high Péclet number cases.

\subsection{Solution of driven flow in a square cavity}

The effectiveness of the proposed scheme in Navier-Stokes flow problems is next investigated in the analysis of a square-cavity flow. Figure 7(a) shows the geometry of the square cavity.

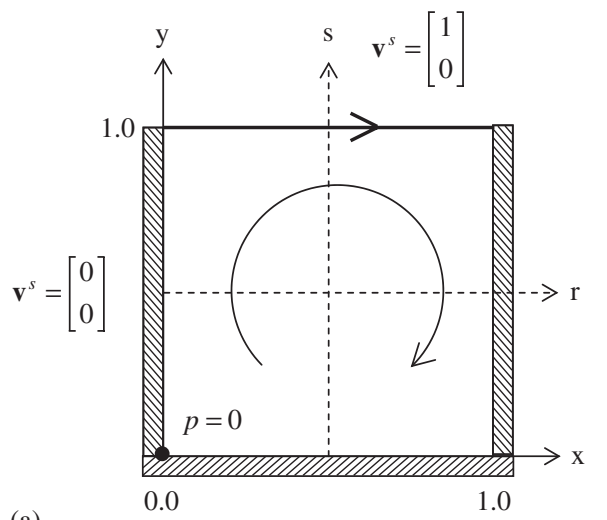

(a)

Figure 7. The lid-driven flow problem in a square cavity: (a) problem definition $(-1 \leqslant r, s \leqslant 1)$; and (b) $20 \times 20$ mesh.

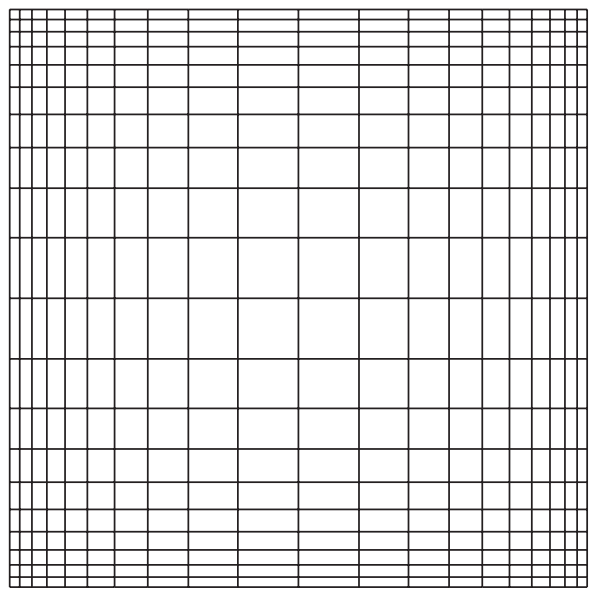

(b) 


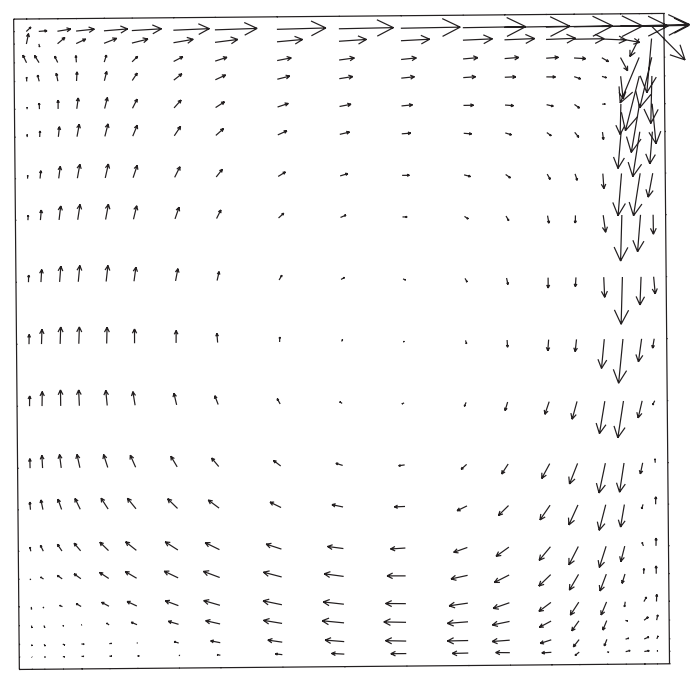

Figure 8 . Velocity distribution inside the cavity obtained with the $10 \times 10$ mesh for $R e=10000$.

The calculation is conducted in the $x, y$ co-ordinate system, and the $r, s$ co-ordinate system along the centre lines are used for the display of the numerical results. The no-slip boundary condition is imposed on the left, lower and right boundaries, while a unit horizontal velocity is prescribed on the upper boundary including the corners. In addition, zero pressure is prescribed at the lower left corner. Five regular meshes that consist of $10 \times 10,20 \times 20,40 \times 40,80 \times 80$ and $160 \times 160$ elements, one of which is shown in Figure 7(b), are used for the solutions. In all meshes used, the grid points are distributed finer near the boundary according to the following equations:

$$
\begin{aligned}
& x(i)=\frac{\mathrm{e}^{(2 \gamma / N)(i-1)}-1}{2\left(\mathrm{e}^{\gamma}-1\right)} L \quad\left(1 \leqslant i \leqslant \frac{N}{2}+1\right) \\
& x(i)=\left\{1-\frac{\mathrm{e}^{(2 \gamma / N)(N+1-i)}-1}{2\left(\mathrm{e}^{\gamma}-1\right)}\right\} L \quad\left(\frac{N}{2}+1 \leqslant i \leqslant N+1\right)
\end{aligned}
$$

where $N$ is the number of elements on a side, $L$ is the length of the side, $i$ is the node number and $\gamma$ represents the parameter for unequal division. The value of $\gamma$ is fixed at 2 for the five meshes.

We first calculate the fluid flow for $R e=10000$ with the $10 \times 10$ mesh. Figure 8 shows the velocity solution inside the cavity. This mesh is, of course, very coarse for this high Reynolds number flow, but still a reasonable velocity distribution is obtained.

Figures 9(a) and (b) show the comparison of our results with the solutions of Ghia et al. [13] for $R e=1000$ and 5000, respectively. It is seen that, as we refine the mesh, the velocity profiles along the centre lines approach those reported by Ghia et al. 

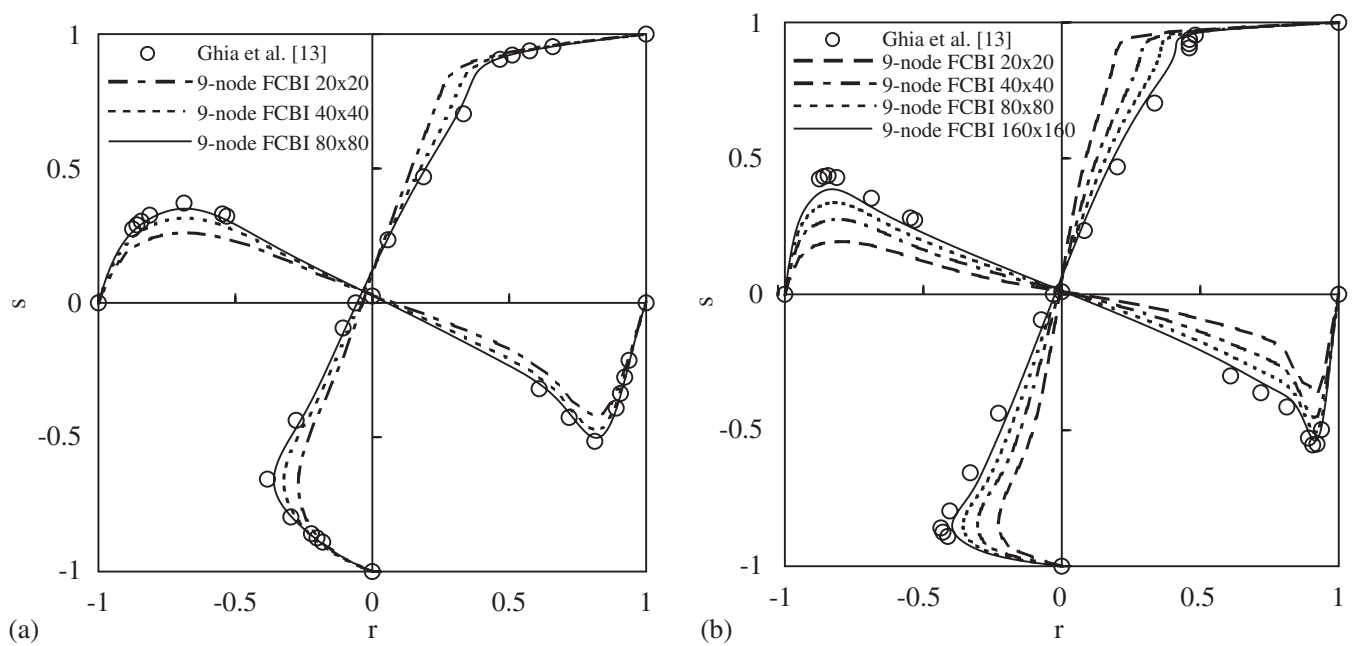

Figure 9. Comparison of vertical and horizontal velocity profiles along the centre lines obtained with four different meshes: (a) $R e=1000$; and (b) $R e=5000$.

\section{CONCLUSIONS}

In this paper we presented the formulation of a nine-node FCBI finite element and some solution results using this element. Of course, further studies of the element formulation and improvements therein are very desirable.

However, the approach of using flow-condition-based interpolations seems to have considerable premise to provide effective fluid flow analysis procedures, in particular when the primary objective is to obtain stable and only reasonable flow solutions using coarse finite element meshes. This is indeed the objective in fluid flow structure interaction problems, where rather coarse meshes to solve for the fluid flow may be sufficient to solve accurately for the structural response. The nine-node element proposed in this paper is a good candidate for error assessment in goal-oriented solutions of a structural response. Here, generally, for the fluid flow, low-order (e.g. four-node) elements would probably be used but higher order (e.g. nine-node) elements might be sometimes employed for an error assessment.

While we considered in this paper only two-dimensional solutions, the concepts employed are also directly applicable to three-dimensional analyses.

\section{APPENDIX A: CONSTRUCTION OF THE TRIAL FUNCTIONS $h_{i}^{v}$}

We consider a one-dimensional advection-diffusion problem, in non-dimensional form, for which the velocity $u$ and the element length $\Delta x(>0)$ are constants, with two fixed values on the boundary ( $\theta_{1}$ and $\theta_{2}$ at $x_{1}$ and $x_{2}$ with $x_{1} \leqslant x \leqslant x_{2}$, respectively). For this problem, the advection-diffusion equation to solve for $\theta$ can be written as

$$
u \frac{\mathrm{d} \theta}{\mathrm{d} x}=\frac{1}{P e} \frac{\mathrm{d}^{2} \theta}{\mathrm{d} x^{2}}
$$


where $P e$ is the Péclet number. The analytical solution of Equation (A1) is

$$
\theta=(1-\lambda) \theta_{1}+\lambda \theta_{2}
$$

with

$$
\lambda=\frac{\mathrm{e}^{P e^{e} \xi}-1}{\mathrm{e}^{P e^{e}}-1}, \quad P e^{e}=P e u \Delta x
$$

In the original FCBI method [8], the above exact solution of the one-dimensional advectiondiffusion equation is directly utilized for the interpolation of the velocity on the element sides. When the flow conditions are introduced into the interpolation, the velocity $\mathbf{v}^{12}$ on the side $1-2$ is obtained with the nodal velocities $\mathbf{v}_{1}$ and $\mathbf{v}_{2}$ as follows:

$$
\mathbf{v}^{12}=\left(1-x^{1}\right) \mathbf{v}_{1}+x^{1} \mathbf{v}_{2}
$$

with

$$
x^{1}=\frac{\mathrm{e}^{R e^{e} \xi}-1}{\mathrm{e}^{R e^{e}}-1}, \quad \operatorname{Re} e^{e}=\operatorname{Re} \overline{\mathbf{v}}^{1} \cdot \Delta \mathbf{x}^{1}
$$

where $\overline{\mathbf{v}}^{1}$ is the average velocity on the side $1-2$, and $\Delta \mathbf{x}^{1}$ is defined as in Equation (24). Of course, if we consider simply the bilinear interpolation, the velocity $\mathbf{v}^{12}$ is interpolated as follows:

$$
\mathbf{v}^{12}=(1-\xi) \mathbf{v}_{1}+\xi \mathbf{v}_{2}
$$

By comparing Equations (A4) and (A6), we notice that the trial functions providing the upwinding effect are established by simply replacing the natural co-ordinate value $\xi$ with $x^{1}$, where the coefficient $x^{1}$ contains the information of fluid flow along the sides. Here, both $\xi$ and $x^{1}$ range from zero to one, and $x^{1}$ approaches $\xi$ when $R e^{e} \rightarrow 0$.

Applying this idea to the quadratic interpolation, we can construct the trial functions $h_{i}^{v}$ shown in Equations (15)-(22). In the biquadratic interpolation over the nine-node element, the velocity $\mathbf{v}_{\|}^{152}$ on the side $1-5-2$ is written as follows:

$$
\mathbf{v}_{\|}^{152}=(1-2 \xi)(1-\xi) \mathbf{v}_{1 \|}+\xi(2 \xi-1) \mathbf{v}_{2 \|}+4 \xi(1-\xi) \mathbf{v}_{5 \|}
$$

Then, by replacing $\xi$ with $x^{15}$ and $x^{52}$, which are, respectively, calculated in Equations (16) and (18), the velocity $\mathbf{v}_{\|}^{152}$ is interpolated on the side by taking account of the flow condition as follows:

$$
\begin{array}{ll}
0 \leqslant \xi \leqslant 0.5: & \mathbf{v}_{\|}^{152}=\left(1-2 x^{15}\right)\left(1-x^{15}\right) \mathbf{v}_{1 \|}+x^{15}\left(2 x^{15}-1\right) \mathbf{v}_{2 \|}+4 x^{15}\left(1-x^{15}\right) \mathbf{v}_{5 \|} \\
0.5 \leqslant \xi \leqslant 1: & \mathbf{v}_{\|}^{152}=\left(1-2 x^{52}\right)\left(1-x^{52}\right) \mathbf{v}_{1 \|}+x^{52}\left(2 x^{52}-1\right) \mathbf{v}_{2 \|}+4 x^{52}\left(1-x^{52}\right) \mathbf{v}_{5 \|}
\end{array}
$$

Note that for $x^{15}$ and $x^{52}$ we have $0 \leqslant x^{15} \leqslant 0.5,0.5 \leqslant x^{52} \leqslant 1$. The velocities $\mathbf{v}_{\|}^{473}$ and $\mathbf{v}_{\|}^{896}$ are also obtained by considering the flow conditions on the corresponding side and centre line in the same way. Finally, the velocity inside the domains 1 and 2 in Figure 2(a) can be expressed by connecting the three velocity values $\mathbf{v}_{\|}^{152}, \mathbf{v}_{\|}^{473}$ and $\mathbf{v}_{\|}^{896}$ with quadratic interpolation as 
follows:

$$
\begin{aligned}
\mathbf{v}_{\|}^{1,2} & =(1-2 \eta)(1-\eta) \mathbf{v}_{\|}^{152}+\eta(2 \eta-1) \mathbf{v}_{\|}^{473}+4 \eta(1-\eta) \mathbf{v}_{\|}^{896} \\
& =h_{i}^{v} \mathbf{v}_{i \|}
\end{aligned}
$$

By the above strategy, we could construct FCBI schemes for various elements in the twoand three-dimensional spaces.

\section{ACKNOWLEDGEMENT}

The work of Haruhiko Kohno was supported by the Japan Society for the Promotion of Science (JSPS) with a JSPS Postdoctoral Fellowship for Research Abroad. We are grateful for this support.

\section{REFERENCES}

1. Bathe KJ (ed.). Computational fluid and solid mechanics 2003. Proceedings of the Second MIT Conference on Computational Fluid and Solid Mechanics. Elsevier: Amsterdam, 2003.

2. Bathe KJ (ed.). Computational fluid and solid mechanics 2005. Proceedings of the Third MIT Conference on Computational Fluid and Solid Mechanics. Elsevier: Amsterdam, 2005.

3. Gresho PM, Sani RL. Incompressible Flow and the Finite Element Method. Wiley: New York, 2000.

4. Drikakis D, Rider W. High-Resolution Methods for Incompressible and Low-Speed Flows. Springer: Berlin, 2005.

5. Grätsch T, Bathe KJ. A posteriori error estimation techniques in practical finite element analysis. Computers and Structures 2005; 83:235-265.

6. Grätsch T, Bathe KJ. Goal-oriented error estimation in the analysis of fluid flows with structural interactions. Computer Methods in Applied Mechanics and Engineering, in press.

7. Bathe KJ, Pontaza JP. A flow-condition-based interpolation mixed finite element procedure for higher Reynolds number fluid flows. Mathematical Models and Methods in Applied Sciences 2002; 12(4):525-539.

8. Bathe KJ, Zhang H. A flow-condition-based interpolation finite element procedure for incompressible fluid flows. Computers and Structures 2002; 80:1267-1277.

9. Kohno H, Bathe KJ. Insight into the flow-condition-based interpolation finite element approach: solution of steady-state advection-diffusion problems. International Journal for Numerical Methods in Engineering 2005; 63:197-217.

10. Kohno H, Bathe KJ. A flow-condition-based interpolation finite element procedure for triangular grids. International Journal for Numerical Methods in Fluids 2005; 49:849-875.

11. Bathe KJ, Zhang H. Finite element developments for general fluid flows with structural interactions. International Journal for Numerical Methods in Engineering 2004; 60:213-232, see also http://www.adina.com

12. Bathe KJ. Finite Element Procedures. Prentice-Hall: Englewood Cliffs, NJ, 1996.

13. Ghia U, Ghia KN, Shin CT. High-Re solutions for incompressible flow using the Navier-Stokes equations and a multigrid method. Journal of Computational Physics 1982; 48:387-411. 